\title{
TÉCNICA DE DOBLE INTUBACIÓN PARA EL TRATAMIENTO DE LA EPÍFORA EN CASOS COMPLICADOS
}

\section{DOUBLE INTUBATION TECHNIQUE FOR THE TREATMENT OF EPIPHORA IN COMPLICATED CASES}

\author{
JUNCEDA-MORENO J ${ }^{1}$, DOS-SANTOS-BERNARDO V ${ }^{2}$, SUÁREZ-SUÁREZ E ${ }^{2}$
}

\begin{abstract}
RESUMEN
Objetivo: Presentar un sistema para corregir quirúrgicamente la epífora ante obstrucciones del sistema lagrimal que se presentan de difícil resolución por alteraciones en fosa nasal, traumatismos o cirugía previa.

Métodos: Se realiza una dacriocistorrinostomía (DCR) por vía externa, colocando una doble intubación concéntrica a nivel de saco lagrimal y fosa nasal introduciendo un elemento de interposición macizo de silicona en otro cilindro hueco, anclado en la abertura del saco. Se practicó la técnica en 27 casos durante los años 2001 y 2002, en pacientes afectados por traumatismos faciales con fractura ósea que compromete el sistema lagrimal (diez casos), con cirugía nasal previa (cuatro casos), con DCR fallida previa en el mismo lado (seis casos), y con DCR previa fallida en ojo contralateral (siete casos).

Resultados: Son valorados según la ausencia de lagrimeo, el vaciamiento del menisco lagrimal, y la permeabilidad objetivable del sistema lagrimal mediante paso forzado de líquido. Los resultados globales indican una tasa de eficacia de la técnica
\end{abstract}

\begin{abstract}
Purpose: The aim of this study is to present a surgical technique used to treat epiphora secondary to difficult cases of lacrimal pathway obstruction after fractures, previous surgery or anatomic abnormalities.

Methods: From January 2001 to December 2002 we treated 27 patients with complicated lacrimal pathway obstruction secondary to fractures (10 cases), previous nasal surgery (4 cases), failure of a previous ipsilateral dacryocystorhinostomy (DCR; 6 cases), or failure of a previous contralateral DCR (7 cases). A DCR through an external approach was performed by placing a double concentric intubation in the lacrimal sac and nasal fossa, with the additional introduction of an interpositional silicone structure within a third hollow tube anchored to the sac entrance.

Results: The results were evaluated according to the absence of tearing, the emptiness of the lacrimal meniscus, and the permeability of the lacrimal system after forced liquid infusion. Globally, the technique was effective in 85 percent of cases. The results were satisfactory in $90 \%$ of cases of pos-
\end{abstract}

Recibido: 6/6/05. Aceptado: 16/2/06

Servicio de Oftalmología. Hospital Valle del Nalón. Asturias. España.

1 Doctor en Medicina.

2 Licenciado en Medicina.

Comunicación presentada en el LXXX Congreso de la S.E.O. (Córdoba 2004).

Correspondencia:

Juan Junceda Moreno

$\mathrm{C} /$. Avenida de Galicia, $13,6{ }^{\circ} \mathrm{C}$

33005 Oviedo (Asturias)

España

E-mail: junceda@yahoo.com 
de un $85 \%$. El análisis por grupos refleja una eficacia del $90 \%$ en pacientes con traumatismo facial previo, del $75 \%$ en pacientes sometidos a cirugía nasal previa, del $83 \%$ en operados de DCR previa en el mismo ojo, y del $71 \%$ en operados de DCR con mal resultado en el ojo contralateral.

Conclusiones: El método presentado nos parece una buena solución en los casos de obstrucciones bajas del sistema lagrimal de pacientes con patología previa, en los que sospechamos un fracaso al enfrentarnos a la cirugía convencional de repermeabilización.

Palabras clave: Vía lagrimal, cirugía de la vía lagrimal, corrección quirúrgica de la epífora, intubación sistema lagrimal. traumatic epiphora, in $75 \%$ of patients with previous surgery, in $83 \%$ of patients with previous ipsilateral DCR, and in $71 \%$ of patients with failed DCR in the contralateral eye.

Conclusions: The double intubation technique is a good option in lower lacrimal system obstructions when conventional techniques have a high index of failure (Arch Soc Esp Oftalmol 2006; 81: 101-106).

Key words: Lacrimal pathway, lacrimal pathway surgery, surgical treatment of epiphora, lacrimal system intubation.

\section{INTRODUCCIÓN}

En ocasiones, las obstrucciones del sistema lagrimal tienen una solución relativamente sencilla, mediante una dacriocistorrinostomía (DCR), por el sistema de abordaje con que el cirujano esté más familiarizado (1). Otras veces podemos encontrar una epífora postquirúrgica después de intentos de repermeación que no han sido eficaces, o bien hallarnos ante patología vecina que compromete el sector vertical de la vía, como traumatismos faciales (2), cirugía maxilofacial, otorrinolaringológica, correcciones de traumatismos, exéresis tumorales (3), limpiezas sinusales, etc. (4), que pueden afectar al conducto lacrimonasal a causa de la patología primitiva o bien alterarlo tras las maniobras quirúrgicas.

En este tipo de casos podemos encontrar una mucosa nasal afectada por la patología primaria. Otras veces la mala calidad o el deterioro de la pituitaria, por enfermedades nasales puede dificultar la realización de uniones quirúrgicas mucomucosas, que deben ser la base de un buen recorrido de nueva formación para el paso de lágrima hacia la fosa nasal.

En una serie de pacientes con obstrucciones complejas de la vía lagrimal se ha realizado una cirugía en la que, mediante abordaje cutáneo, se practica una DCR en la cual se asocia como elemento de interposición un cilindro hueco de silicona fijado en una ventana practicada en el saco lagrimal, en el que se introduce un doble cilindro macizo que actúa como tutor de la cicatrización en la fase postoperatoria, a fin de comprobar si el resultado obtenido es más favorable que con la DCR convencional, tutorizada o no.

\section{SUJETOS, MATERIAL Y MÉTODOS}

Se ha realizado un estudio de la cirugía efectuada en 27 vías lagrimales obstruidas en su segmento o porción vertical, cuyas causas son recogidas en la tabla I. Del total de pacientes, seis casos fueron intervenidos por DCR fallida en el mismo lado, siete habían sufrido una DCR en el otro lado que resultó fallida, diez estaban afectados por un traumatismo afectando a la porción vertical del sistema lagrimal, y cuatro habían padecido una patología vecina, inflamatoria u oncológica tributaria de cirugía ORL, que afectó a su sistema lagrimal.

Fueron descartados aquellos pacientes en los que el sistema lagrimal estaba afectado en su porción horizontal por el mismo procedimiento que generó la obstrucción primitiva, y que fueron tributarios de otro tipo de cirugía. En todos los casos se realizó dacriocistografía previa a la cirugía.

Tabla I. Patología tributaria de la cirugía y número de casos

\begin{tabular}{lc}
\hline Diagnóstico & N. $^{\text {o de casos }}$ \\
\hline DCR previa fallida ipsilateral & 6 \\
DCR previa fallida contralateral & 7 \\
Traumatismo afectando sistema lagrimal & 10 \\
Cirugía ORL previa (nasal, sinusal) & 4 \\
\hline \hline
\end{tabular}

DCR: dacriocistorrinostomía. 
En los pacientes seleccionados se realizó una DCR por abordaje cutáneo, disecando la rama ascendente del maxilar superior y practicando una osteotomía ovoidea de $10 \times 7 \mathrm{~mm}$ de eje mayor colocado hacia abajo y la altura aproximada del párpado inferior. En los casos en los que se había realizado cirugía repermeabilizadora previa se amplió en los casos necesarios la osteotomía y se limpió el tejido fibroso que se encontró obstaculizando la comunicación saco lagrimal-pituitaria. Una vez analizadas las estructuras y localizadas las paredes del saco lagrimal, se procedió a resecar parte de la pared interna del mismo, en forma cuadrangular y prolongando los cortes verticales hacia abajo y atrás a fin de obtener un suficiente plano mucoso para obtener una adecuada superficie que permitiera anclar el sistema de permeación diseñado.

Realizamos un orificio cuadrangular en el saco lagrimal, obviamente cuando existe suficiente porción de saco como para ello, como por ejemplo, cuando el problema se ha generado por cirugía nasal (ORL) o por haber encontrado una DCR fallida en ojo contralateral.

Cuando nos enfrentamos a una re-DCR en la cual ya no tenemos suficiente material sacular, la apertura cuadrangular queda reducida a un túnel o embudo mucoso que, retrógradamente, lleva al conducto común, que es por donde vienen los elementos macizos de silicona, En tal caso unimos el tubo excéntrico(grueso) a la parte más ancha (o más nasal) del «embudo» citado. Como es lógico, en estos casos no se puede disecar el saco lagrimal, sino utilizar el remanente de mucosa que pueda existir.

Se introdujo desde la ventana nasal ipsilateral hacia la ventana del saco a través de la osteotomía un tubo sintético hueco de $15 \mathrm{G}$ (sonda sintética 06004419, IZASA S.A., Barcelona, España); en dicho tubo se introduce posteriormente un sistema de intubación bicanalicular de silicona maciza $25 \mathrm{G}$ (Canaliculus Intubation Set 8590450, Medtronic Solan, Jacksonville, FL, USA) (este sistema es enhebrado de forma que entra por el orificio proximal y sale por el distal del tubo hueco introducido en primera instancia). El orificio proximal del tubo hueco es anclado al saco lagrimal con doble sutura de seda 6-0, en la porción anterior y en la posterior de la ventana practicada en el saco lagrimal (fig. 1). El orificio distal del tubo hueco es recortado a la altura de la ventana nasal y los tubos de silicona bicanaliculares son fijados a la mejilla con esparadrapo (fig. 2). La retirada del tubo hueco se realiza

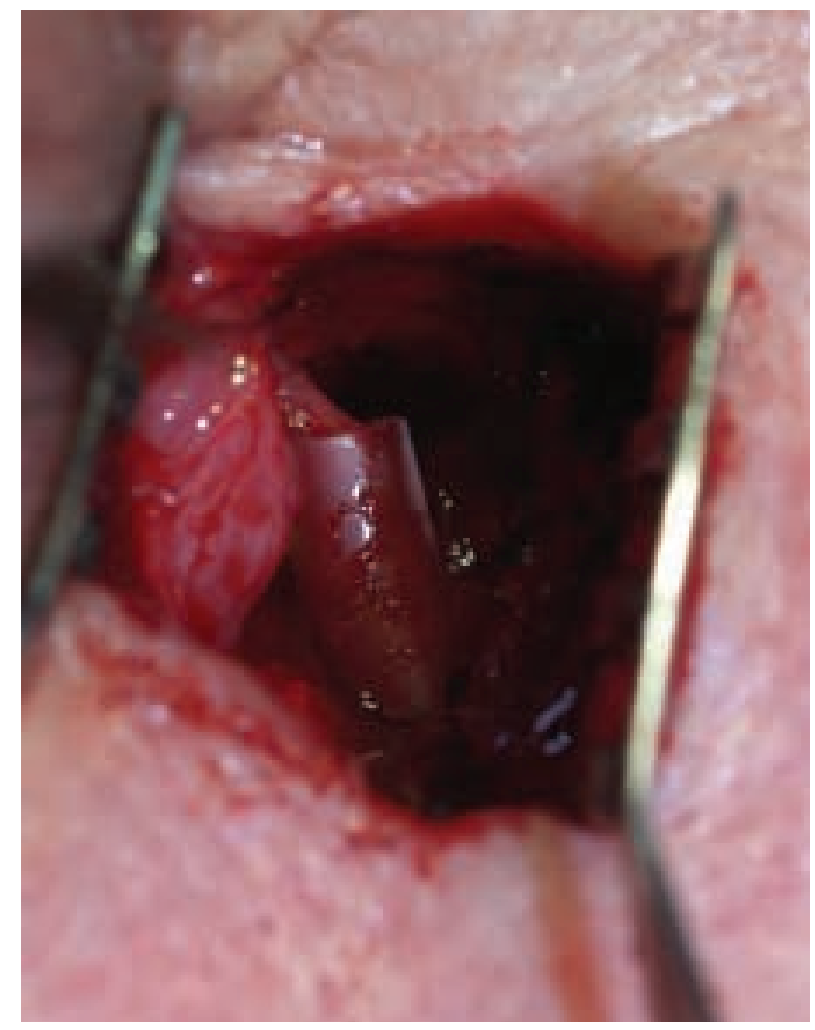

Fig. 1: Imagen intraoperatoria del tubo externo del sistema de doble intubación, fijado en la zona del saco lagrimal.

en consultorio a las 2 semanas y la retirada de la intubación bicanalicular se realiza en consultorio a las 3 semanas. Todos los pacientes fueron intervenidos bajo anestesia general, dado que no era posible preveer la dificultad y/o duración de la cirugía en estos casos.

Se realizó un estudio descriptivo longitudinal de la muestra de forma que las variables cuantitativas se describen con la media y los percentiles 25 y 75; y las cualitativas por la distribución de frecuencias relativas. Posteriormente, se realizó un análisis bivariante buscando la relación de las variables de edad, sexo y motivo de la cirugía con aquellas relacionadas con los resultados. Se utilizaron las pruebas de chi-cuadrado, prueba exacta de Fisher con dos colas para variables cualitativas y la prueba de $\mathrm{U}$ de Mann-Whitney para variables cuantitativas. Se definió un nivel de significación estadística del 5\%, presentando los intervalos de confianza al $95 \%$ entre paréntesis. Todos los análisis se llevaron a cabo con el paquete estadístico SPSS v.8.0 para Windows (SPSS Inc., Chicago, ILL, USA). 


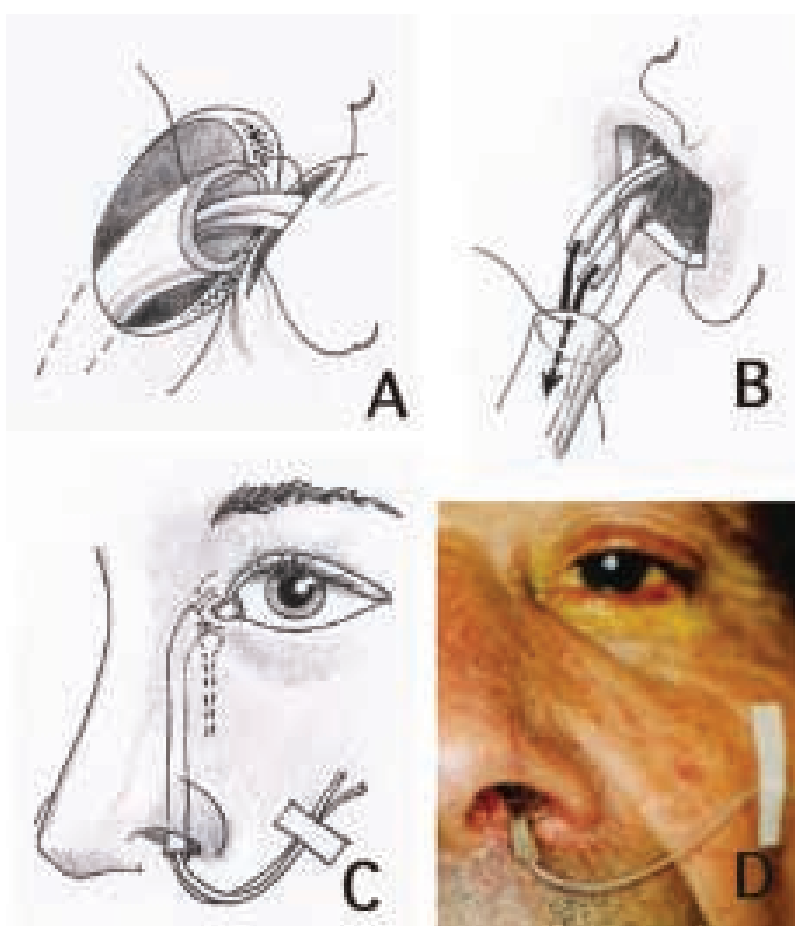

Fig. 2: A) Esquema de la colocación del dispositivo de doble intubación, visto desde la hendidura palpebral. B) Esquema análogo, visto desde fosa nasal. C) Reconstrucción teórica del sistema. Los puntos interrumpidos indican la situación del saco lagrimal. D) Aspecto a las 2 semanas de la intervención.

\section{RESULTADOS}

Se valoraron según la ausencia de lagrimeo, el vaciamiento del menisco lagrimal (retención de lágrima), y la permeabilidad objetivable del sistema lagrimal mediante paso forzado de líquido (siringa- ción). Los resultados globales indican una tasa de eficacia de la técnica de un $85 \%$. La valoración por grupos refleja una eficacia del $90 \%$ de la técnica en pacientes con traumatismo facial previo, del $75 \%$ en pacientes sometidos a cirugía nasal previa, del $83 \%$ en operados de DCR previa en el mismo ojo, y del $71 \%$ en operados de DCR con mal resultado en el otro ojo.

En los pacientes estudiados el 69,2\% $(49,6-88,9)$ eran varones. Su edad media era de 55,7 años $(50,6-$ 60,8). El 25\% tenían menos de 46,7 años y otro $25 \%$ tenía más de 66 años. En el 34,6\% (14,4-54,8) de los casos la DCR estuvo justificada por la existencia de una fractura ósea, en el 26,9\% (7,9-45,9) por DCR previa fallida en ojo contralateral, en el $23,1 \%(9-43,6)$ por DCR previa fallida del mismo ojo y en un $15,4 \%(4,4-34,9)$ por cirugía nasal previa.

En relación con los resultados globales de la técnica (considerando conjuntamente la ausencia de lagrimeo, el no vaciamiento del menisco lagrimal y la permeabilidad objetivable del sistema lagrimal), ésta fue efectiva en un 80,8\% $(60,6-93,4)$ de los casos. En un 19,2\% de los casos $(6,6-39,4)$ no había vaciamiento del menisco lagrimal mientras que en un $15,4 \%(4,4-34,9)$ había persistencia del lagrimeo y en otro $15,4 \%$ la permeabilidad no era objetivable. No se presentaron otro tipo de complicaciones. En relación con la influencia de las diferentes variables en los resultados, los datos se recogen en la tabla II. De esta tabla se desprende que los pacientes en los que la técnica es efectiva tienen una menor edad media que aquellos en los que no es efectiva, alcanzándose significación estadística en relación con la permeabilidad forzada. No se encontraron diferencias estadísticamente significativas en

Tabla II. Diferencias en efectividad de la técnica en función de edad, sexo y motivo de la cirugía

\begin{tabular}{|c|c|c|c|c|c|c|c|c|c|c|c|c|}
\hline \multirow{2}{*}{$\begin{array}{l}\text { Variables de resultado } \\
\text { Otras Variables }\end{array}$} & \multicolumn{3}{|c|}{ Efectividad global } & \multicolumn{3}{|c|}{ Epífora residual } & \multicolumn{3}{|c|}{ Retención de lágrima } & \multicolumn{3}{|c|}{ Permeabilidad } \\
\hline & No & Sí & $\mathrm{p}$-valor & Sí & No & $\mathrm{p}$-valor & Sí & No & p-valor & Sí & No & p-valor \\
\hline $\begin{array}{l}\text { Edad } \\
\quad(\text { media y E.S.) }\end{array}$ & $\begin{array}{l}64,4 \\
(2,6)\end{array}$ & $\begin{array}{l}53,6 \\
(2,8)\end{array}$ & 0,08 & $\begin{array}{l}65,2 \\
(3,2)\end{array}$ & $\begin{array}{l}53,9 \\
(2,7)\end{array}$ & 0,088 & $\begin{array}{l}64,4 \\
(2,6)\end{array}$ & $\begin{array}{l}53,6 \\
(2,8)\end{array}$ & 0,084 & $\begin{array}{l}53,8 \\
(2,7)\end{array}$ & $\begin{array}{l}66 \\
(2,7)\end{array}$ & 0,046 \\
\hline \multicolumn{13}{|l|}{ Sexo } \\
\hline Varones & $16,7 \%$ & $83,3 \%$ & n.s. & $11,1 \%$ & $88,9 \%$ & n.s. & $16,7 \%$ & $83,3 \%$ & n.s. & $88,9 \%$ & $11,1 \%$ & n.s. \\
\hline Mujeres & $25 \%$ & $75 \%$ & & $25 \%$ & $75 \%$ & & $25 \%$ & $75 \%$ & & $75 \%$ & $25 \%$ & \\
\hline \multicolumn{13}{|l|}{ Causa } \\
\hline Fractura ósea & $11,1 \%$ & $88,9 \%$ & n.s. & $11,1 \%$ & $88,9 \%$ & n.s. & $11,1 \%$ & $88,9 \%$ & n.s. & $100 \%$ & 0 & n.s. \\
\hline Cirugía nasal & $25 \%$ & $75 \%$ & & $25 \%$ & $75 \%$ & & $25 \%$ & $75 \%$ & & $75 \%$ & $25 \%$ & \\
\hline DCR mismo ojo & $16,7 \%$ & $83,3 \%$ & & $16,7 \%$ & $83,3 \%$ & & $16,7 \%$ & $83,3 \%$ & & $83,3 \%$ & $16,7 \%$ & \\
\hline DCR contralateral & $28,6 \%$ & $71,4 \%$ & & $14,3 \%$ & $85,7 \%$ & & $28,6 \%$ & $71,4 \%$ & & $71,4 \%$ & $28,6 \%$ & \\
\hline
\end{tabular}

E.S.: Error estándar de la media; n.s.: No significación estadística. 
la efectividad de la técnica (a nivel global o en cada uno de sus componentes) en función del sexo o del motivo de la cirugía.

\section{DISCUSIÓN}

En términos generales, cuando se padece una epífora por obstrucción en el conducto lacrimonasal parece razonable pensar que la cirugía repermeabilizadora convencional puede ser eficaz para restablecer la continuidad de la vía lagrimal, dado que ofrece una tasa de éxito que oscila entre el 87 y el 97 por ciento, según los autores $(5,6)$, obteniéndose una adecuada funcionalidad (7) con una mejora en la calidad de vida del paciente.

Cuando hay que tratar a un paciente con afectación importante del conducto lacrimonasal o de otra zona del sector vertical de la vía lagrimal, se podría pensar que la cirugía repermeabilizadora va a ser difícil si el origen del problema está en un traumatismo o en una cirugía previa (8).

Otras veces existen casos de pacientes en los que la cirugía repermeabilizadora ha fracasado por diversos motivos. En ocasiones, por el mal estado de las mucosas al practicar la unión de colgajos. También puede impedir el éxito de la cirugía una bizarra cicatrización que rellena la zona intervenida de elementos fibrosos, y ocasionalmente un cierre inesperado de la osteotomía efectuada (9). O pueden existir otros motivos, como una escasa apertura y comunicación con la mucosa nasal del saco lagrimal durante la cirugía realizada (10). En algunas ocasiones, puede plantear problemas durante la cirugía el manejo de la fosa nasal, con la que no está habitualmente familiarizado el cirujano, dando lugar a problemas para la realización de colgajos mucosos cuando esta zona no está anatómicamente y/o funcionalmente en buen estado (11).

Como es natural, cuando ha sucedido algo parecido y el paciente requiere una reintervención, hay que estudiar las causas del fracaso y valorar adecuadamente los pasos realizados para evitar que se repita. Lo mismo sucede cuando es necesario, en casos de afectación bilateral, intervenir el lado contralateral.

Ante algunos de estos casos, han existido diversos procedimientos que tratan de evitar el fracaso en estos casos de teóricamente difícil solución. Algunos autores aconsejan la realización de cistectomía o dacriocistectomía en un segundo tiempo para solucionar el problema (12). En otras ocasiones se opta por la tutorización, con material metálico o sintético (13). Algunos cirujanos prefieren la utilización de medicamentos antiblásticos en impregnación directa, como la mitomicina C (14), algunos se decantan por el empleo de tubos de Jones (15), y otros incluso por la revisión transcanalicular con láser del área intervenida (16).

En cualquier caso, al existir diferentes métodos para intentar solucionar el problema, parece que ninguno de ellos ofrece una garantía suficientemente amplia como para imponerse a los demás, si bien las series de casos en los que se han empleado cada uno de las métodos propuestos son cortas, excepción hecha de la colocación del tubo de L. Jones.

Nosotros hemos utilizado una técnica que nació ante la dificultad de encontrar elementos mucosos para realizar un adecuado túnel o canal de paso de lágrima durante una DCR en uno de estos casos, afectado por una fractura de LeFort. A partir de este momento la hemos empleado con asiduidad cuando el caso al que nos enfrentamos «a priori» nos parece de difícil resolución o bien cuando, intraoperatoriamente encontramos dificultades que van a impedir la adecuada reconstrucción del área intervenida.

Hurwitz y cols. emplearon un sistema similar, basándose en la técnica quirúrgica propuesta por Iliff para la DCR (17), si bien el tubo excéntrico era más corto, la retirada escalonada de elementos es diferente y no han sido publicados sus resultados. Los nuestros indican que este tipo de método es eficaz para corregir el problema en más del $80 \%$ de los casos, por lo que se propone como solución para aquellos casos en los que presumiblemente podemos encontrarnos con dificultades a la hora de repermeabilizar un sistema lagrimal obstruido.

\section{BIBLIOGRAFÍA}

1. Bartley GB. Acquired lacrimal drainage obstruction: an etiologic classification system, case reports, and a review of the literature. Part 2. Ophthal Plast Reconstr Surg 1992; 8: 243-249.

2. Osguthorpe JD, Hoang G. Nasolacrimal injuries. Evaluation and management. Otolaryngol Clin North Am 1991; 24: $59-78$.

3. Powell JB 2nd. Nasolacriminal dysfunction. Laryngoscope 1983; 93: 498-515.

4. Meyers AD, Hawes MJ. Nasolacrimal obstruction after inferior meatus nasal antrostomy. Arch Otolaryngol Head Neck Surg 1991; 117: 208-211.

5. Junceda J, Alvarez J, Coronel J. Dacriocistorrinostomía versus Dacriocistorrinostomía tutorizada. Arch Soc Esp Oftalmol 1995; 69: 63-70. 
6. Zolli CL, Shannon GM. Dacryocystorhinostomy: a review of 119 cases. Ophthalmic Surg 1982; 13: 905-910.

7. Delaney $Y M$, Khooshabeh $R$. Fluorescein transit test time and symptomatic outcomes after external dacryocystorhinostomy. Ophthal Plast Reconstr Surg 2002; 18: 281-284.

8. Kashkouli MB, Parvaresh M, Modarreszadeh M, Hashemi $M$, Beigi B. Factors affecting the success of external dacryocystorhinostomy. Orbit 2003; 22: 247-255.

9. Jordan DR. Standard external dacryocystorhinostomy. In: Mauriello JA Jr. Unfavourable results of eyelid and lacrimal surgery: prevention and management. Boston : Butterworth-Heinemann; 2000; 519-549.

10. Linberg JV. Complicaciones de la cirugía lagrimal. In: Krupin T, Kolker AE, Rosenberg LF. Complicaciones en oftalmología quirúrgica. $2 .{ }^{a}$ Ed. Madrid: Harcourt; 2000 . 255-270.

11. Gonnering RS. Dacryocystorhinostomy and conjunctivodacryocystorhinostomy. In: Dortzbach RK. Ophthalmic plastic surgery. Prevention and management of complications. New York: Raven Press; 1994; 237-250.
12. Boynton JR, Anawis MA. Role of dacryocystectomy in the management of failed dacryocystorhinostomy associated with chronic dacryocystitis. Ophthalmic Surg Lasers 1996; 27: 133-136.

13. Dailey RA, Sigler SC, Saulny SM. Surgery of the lacrimal system. In: Spaeth GL. Ophthalmic surgery. Principles and practice. $3{ }^{a}{ }^{2}$ Ed. Philadelphia: Saunders; 2003; 509. 525.

14. Liao SL, Kao SC, Tseng JH, Chen MS, Hou PK. Results of intraoperative mitomycin C application in dacryocystorhinostomy. Br J Ophthalmol 2000; 84: 903-906.

15. Zilelioglu G, Gunduz K. Conjunctivodacryocystorhinostomy with Jones tube. A 10-year study. Doc Ophthalmol 1996; 92: 97-105.

16. Woo KI, Moon SH, Kim YD. Transcanalicular laser-assisted revision of failed dacryocystorhinostomy. Ophthalmic Surg Lasers 1998; 29: 451-455.

17. Hurwitz JJ, Archer KF, Gruss JS. Double stent intubations in difficult post-traumatic dacryocystorhinostomy. Ophthalmic Surg 1988; 19: 33-36. 